\title{
Chromosomal aberrations after induced pluripotent stem cells reprogramming
}

\author{
Isadora May Vaz ${ }^{1,4}$ (D), Tamara Borgonovo ${ }^{1,4}$, Tais Hanae Kasai-Brunswick ${ }^{2,3}$, Danúbia Silva dos Santos ${ }^{2}$, \\ Fernanda Cristina Paccola Mesquita ${ }^{2}$, Juliana Ferreira Vasques ${ }^{2,4}$, Fernanda Gubert ${ }^{4,5}$, \\ Carmen Lúcia Kuniyoshi Rebelatto ${ }^{1,4}$, Alexandra Cristina Senegaglia ${ }^{1,4}$ and Paulo Roberto Slud Brofman ${ }^{1,4}$ \\ 'Pontificia Universidade Católica do Paraná, Escola de Medicina, Núcleo de Tecnologia Celular, Curitiba, \\ PR, Brazil. \\ ${ }^{2}$ Universidade Federal do Rio de Janeiro, Instituto de Biofisica Carlos Chagas Filho, Rio de Janeiro, \\ RJ, Brazil. \\ ${ }^{3}$ Universidade Federal do Rio de Janeiro, Centro Nacional de Biologia Estrutural e Bioimagem, \\ Rio de Janeiro, RJ, Brazil. \\ ${ }^{4}$ Instituto Nacional de Ciência e Tecnologia em Medicina Regenerativa, Rio de Janeiro, RJ, Brazil. \\ Instituto de Ciências Biomédicas, Rio de Janeiro, RJ, Brazil.
}

\begin{abstract}
Induced pluripotent stem cells (iPSCs) are generated from adult cells that have been reprogrammed to pluripotency. However, in vitro cultivation and genetic reprogramming increase genetic instability, which could result in chromosomal abnormalities. Maintenance of genetic stability after reprogramming is required for possible experimental and clinical applications. The aim of this study was to analyze chromosomal alterations by using the G-banding karyotyping method applied to 97 samples from 38 iPSC cell lines generated from peripheral blood or Wharton's jelly. Samples from patients with long QT syndrome, Jervell and Lange-Nielsen syndrome and amyotrophic lateral sclerosis and from normal individuals revealed the following chromosomal alterations: acentric fragments, chromosomal fusions, premature centromere divisions, double minutes, radial figures, ring chromosomes, polyploidies, inversions and trisomies. An analysis of two samples generated from Wharton's jelly before and after reprogramming showed that abnormal clones can emerge or be selected and generate an altered lineage. IPSC lines may show clonal and nonclonal chromosomal aberrations in several passages (from P6 to P34), but these aberrations are more common in later passages. Many important chromosomal aberrations were detected, showing that G-banding is very useful for evaluating genetic instability with important repercussions for the application of iPSC lines.
\end{abstract}

Keywords: Genetic instability, cytogenetics, iPSC, chromosomal aberration.

Received: May 17, 2020; Accepted: June 15, 2021.

\section{Introduction}

Induced pluripotent stem cells (iPSCs) have attracted attention because of their great potential for application in several areas of cell therapy. These cells can differentiate into any cell in the germ layers (endoderm, mesoderm or ectoderm). Due to the widespread use of iPSCs as a model to study diseases, mainly to understand their mechanisms, and their possible uses in regenerative medicine, iPSCs are believed to have a promising future (Ambrosio et al., 2015). iPSCs are currently being used for the study of many congenital syndromes, such as long QT syndrome (LQTS), which was the first heart disease model established using this cell type (Moretti et al., 2010).

Despite advances in stem cell culture conditions, an important issue that needs to be considered is the possibility of an increase in genomic instability. This could explain, at least in part, the potential of these cells to become tumorigenic (Catalina et al., 2007). In addition, altered karyotypes interfere

Send correspondence to Isadora May Vaz. Pontifícia Universidade Católica do Paraná, Rua Imaculada Conceição, 1155, Prado Velho, Curitiba, PR, Brazil. E-mail: isavaz92@yahoo.com.br not only with clinical applications but also with experimental results, such as in drug tests, because their sensitivity to drugs may be affected by aberrant expression patterns of the cells (Ben-David et al., 2014). The study of aneuploidies (numerical chromosomal alterations) is an important aim for guaranteeing the quality of these cells, especially when cells derived from somatic cells from patients have aged (Garcia-Martinez et $a l ., 2016)$. In disease models, altered cells do not always reproduce the same characteristics as the normal cells that give rise to them (Ben-David et al., 2014), therefore, they cannot be used as a model. Thus, it is essential to guarantee the quality, safety and traceability of the cells.

The genomic integrity of the cells can be evaluated by a variety of techniques, including karyotype analysis (Mayshar et al., 2010; Ben-David et al., 2014). Many karyotypic abnormalities have been repeatedly reported in human iPSCs, such as trisomy 12 (Taapken et al., 2011; Ben-David et al., 2014; Lamm et al., 2016) and trisomy 17 (Ben-David and Benvenisty, 2011; Taapken et al., 2011; Dewhurst et al., 2014).

Through the G-banded technique, it is possible to detect numerical and structural anomalies, such as translocations and inversions. In addition to these advantages, this technique has a low cost and can detect low-level mosaicism (usually 20 
metaphases are observed, which is likely to detect a result when more than $5 \%, 1$ in 20, of cells present a chromosomal aberration) (Ben-David and Benvenisty, 2012). However, one of the limitations of the G-banding method is the impossibility of detecting some small alterations, thus complementary methods are sometimes required. The aim of this study was to analyze the genomic integrity of iPSCs using G-banded chromosomal karyotype analysis before and after genetic reprogramming and long-term cultivation to describe chromosomal instability in iPSCs from individuals with diseases associated with genetic mutations and in iPSCs of healthy individuals.

\section{Materials and Methods}

\section{Patients and samples}

The samples were separated into two groups: Group 1 was composed of samples from 21 patients with diseases associated with genetic mutations. These patients were diagnosed with long QT syndrome (LQTS) type 1 (mean age 22.2 years), LQTS type 2 (mean age 34.4 years), Jervell and Lange-Nielsen syndrome (JLNS) (mean age 3.5 years) or amyotrophic lateral sclerosis (ALS) (mean age 53.6 years), in which the source of the cells was peripheral blood. Group 2 was composed of samples from 17 healthy individuals, and the cells were obtained from peripheral blood (mean age 29.1 years) or Wharton's jelly (a tissue present around the umbilical blood vessels).

\section{Cell culture and G-band karyotyping protocol}

The human iPSC cell lines were generated from peripheral blood or from Wharton's jelly at Universidade Federal do Rio de Janeiro. To generate the cell lines, a CytoTune ${ }^{\mathrm{TM}}$ 2.0- Sendai Reprogramming Kit containing OCT-3/4, K1f4, Sox 2 and cMyc as reprogramming factors was used. Expression of pluripotency markers was determined by RT-PCR and flow cytometry (BD Accuri C6, BD FACSAria II and Flow Jo software version 1). The reprogramming method used has already been validated (Kasai-Brunswick et al., 2018; Mesquita et al., 2019; Gubert et al., 2019a; Gubert et al., 2019b).

Confluence was evaluated before chromosomes were harvested. The ideal cytogenetic harvest condition was when the culture reached $60 \%$ to $80 \%$ confluence. Then, the cells were incubated at $37^{\circ} \mathrm{C}$ with $0.1 \mu \mathrm{g} / \mathrm{ml}$ KaryoMAX ${ }^{\circledR}$ Colcemid $^{\mathrm{TM}}$ (Life Technologies) for one hour, trypsinized, added to $6 \mathrm{ml}$ of hypotonic solution (0.075 $\mathrm{M} \mathrm{KCl}$ with HEPES) and fixed with methanol-acetic acid solution (3:1; Merck). The slides were placed in a water bath at $60^{\circ} \mathrm{C}$, and drops of the cell suspension were placed on slides. Chromosomal preparations were submitted to the G-banding method using trypsin and Giemsa-staining. This protocol was based on protocols by Moralli et al. (2011) with modifications and Borgonovo et al. (2014) with modifications.

\section{Analysis and interpretation}

The metaphases were digitally captured with a Leica DM Microscope (DM2000). LUCIA-KARYO (Laboratory Universal Computer Image Analysis LIM - Laboratory Imaging s.r.o) was used for the analysis. Whenever possible, 20 metaphases were analyzed. To be considered a clone, the same structural aberration or gain of the same chromosome had to be present in at least two metaphase cells, and the loss of a a chromosome must have been detected in at least three cells (McGowan-Jordan et al., 2016).

The statistical analysis was performed with IBM SPSS Statistics v.20.0 software (Armonk, NY; IBM Corp). The results were evaluated using the median, minimum and maximum values. Frequencies and percentages were defined for categorical variables. Fisher's exact test was used to analyze the association between two categorical variables. Values of $\mathrm{p}<0.05$ indicated statistical significance.

\section{Results}

Ninety-seven samples from 38 iPSC lines between the $3 \mathrm{rd}$ and 34th passages were evaluated. A total of 632 metaphases were analyzed. Samples without metaphase accounted for $10.8 \%$ of all analyzed cases. The majority (71\%) of samples showed a normal karyotype (excluding samples with less than 20 normal metaphases or those without metaphases).

The samples were separated into two groups. The first group was the disease group including cells from patients diagnosed with LQTS type $1(n=6)$, LQTS type $2(n=8)$, JLNS $(n=2), \operatorname{ALS}(n=3)$ and undetermined diagnoses of LQTS $(n=2)$, totaling 21 samples. The second group was the nondisease group with 17 samples.

Cytogenetic analysis demonstrated the presence of the following structural chromosomal alterations: acentric fragments, chromosomal fusions, premature centromere divisions, double minutes (dmin), radial figures, ring chromosomes, inversions, additional material and marker chromosomes, and the following numerical chromosomal alterations: polyploidies and trisomies (Figure 1). In the disease group, structural alterations were only observed in LQTS type 2 samples, and in the nondisease group, the samples derived from Wharton's jelly presented both numerical and structural alterations, while those derived from blood presented only structural alterations.

\section{Nondisease and disease groups}

Both the disease and nondisease groups presented alterations, with no significant difference between them ( $p=0.139$, Fisher's exact test).

The following percentages of altered samples were observed for each disease in the disease group: LQTS $(n=14$, frequency $21.4 \%)$, JLNS ( $n=2$, frequency $0 \%)$ and ALS $(n=3$, frequency $0 \%$ ); all of the cells were derived from peripheral blood.

Among the total samples from each group, $15.8 \%$ were altered in the disease group and $40 \%$ were altered in the nondisease group. Considering only the samples derived from peripheral blood in the nondisease group, $16.7 \%(n=6)$ were altered, while $62.5 \%(n=8)$ of samples obtained from Wharton's jelly were altered.

Karyotype analysis in the different groups is shown in Table 1.

\section{Differences based on iPSC sources}

The difference between the samples that presented clonal alterations and those that presented normal cytogenetics 

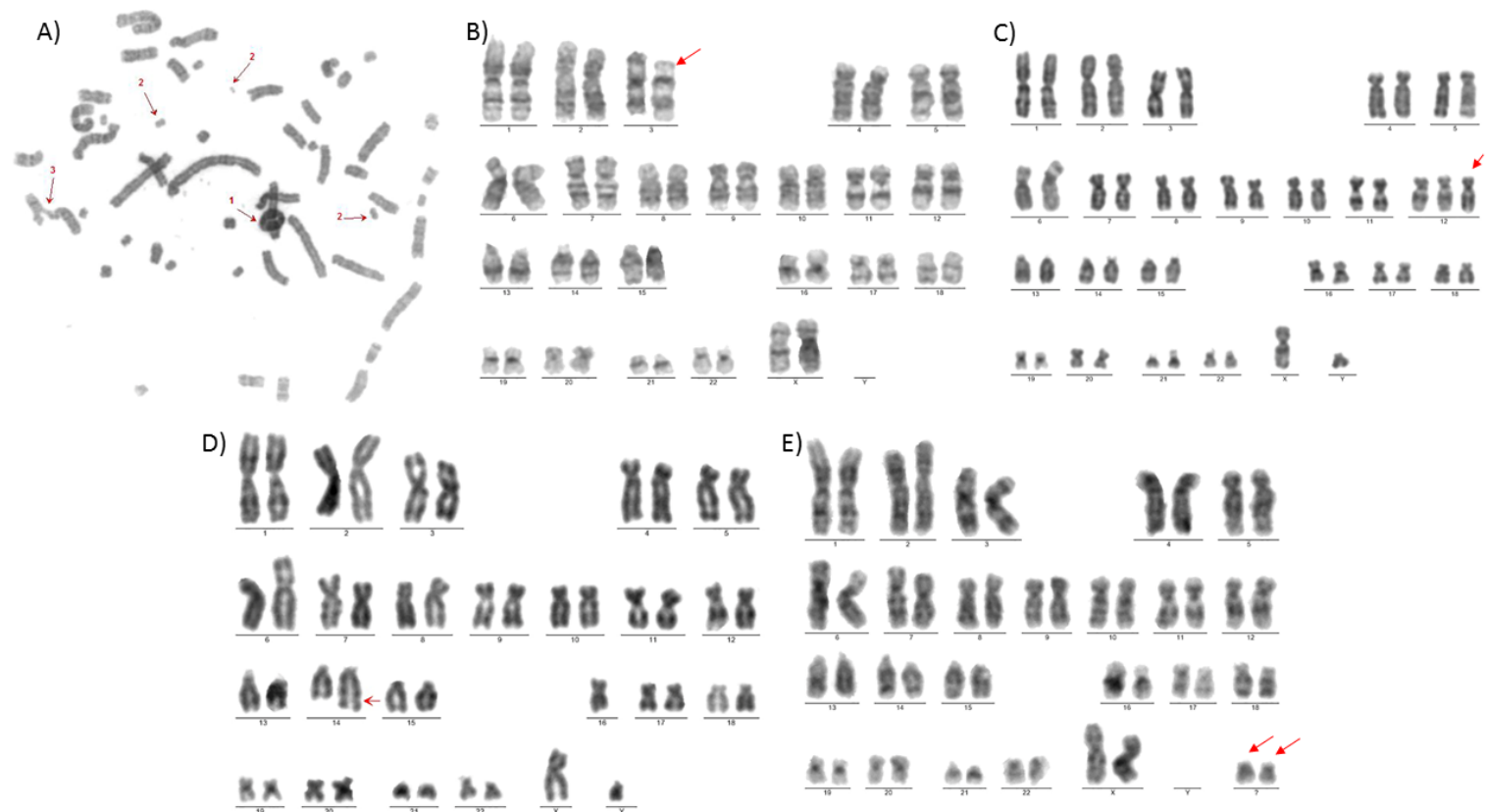

Figure 1 - Cytogenetic analysis. A) Metaphase with ring chromosome (1), double minutes (2) and figure (3) (Sample IP028). B) Karyogram showing chromosome 3 inversion (Sample IP037). C) Karyogram showing chromosome 12 trisomy (Sample IP044). D) Karyogram showing additional material on the long arm of chromosome 14 (Sample IP056). E) Karyogram showing two marker chromosomes (Sample IP039).

Table 1 - Karyotype analysis in the different groups.

\begin{tabular}{|c|c|c|c|c|}
\hline Patient number & Passage & Disease & Source & Karyotype \\
\hline IP020 & 3 & LQTS type 1 & Peripheral blood & Without metaphases \\
\hline IP023 & 4 & LQTS type 1 & Peripheral blood & $46, \mathrm{XX}[20]$ \\
\hline IP031 & 3 & LQTS type 1 & Peripheral blood & $46, X X[20]$ \\
\hline IP033 & 5 & LQTS type 1 & Peripheral blood & $46, \mathrm{XX}[20]$ \\
\hline IP036 & 3 & LQTS type 1 & Peripheral blood & $46, X X[20]$ \\
\hline IP050 & 6 & LQTS type 1 & Peripheral blood & $46, X X[20]$ \\
\hline IP016 & 11 & LQTS type 2 & Peripheral blood & Without metaphases \\
\hline IP017 & 11 & LQTS type 2 & Peripheral blood & $46, X Y[3]$ \\
\hline IP025 & 16 & LQTS type 2 & Peripheral blood & $46, \mathrm{XX}[20]$ \\
\hline IP028 & 15 & LQTS type 2 & Peripheral blood & $36 \sim 45, \mathrm{XY}, \sim 6 \mathrm{dmin},+\mathrm{r} 1,+\mathrm{r} 2[6] / 46, \mathrm{XY}[14]$ \\
\hline IP037 & 6 & LQTS type 2 & Peripheral blood & $46, X X, \operatorname{inv}(3)(p 24 q 2 ? 9)[6] / 46, X X[14]$ \\
\hline IP039 & 28 & LQTS type 2 & Peripheral blood & $37 \sim 45, \mathrm{XX}[9] / 42 \sim 48, \mathrm{XX},+\operatorname{mar} 1,+\operatorname{mar} 2[11]$ \\
\hline IP040 & 6 & LQTS type 2 & Peripheral blood & $46, X Y[20]$ \\
\hline IP051 & 7 & LQTS type 2 & Peripheral blood & $46, \mathrm{XX}[21]$ \\
\hline IP026 & 5 & LQTS undetermined & Peripheral blood & $46, X Y[20]$ \\
\hline IP019 & 3 & LQTS undetermined & Peripheral blood & $46, X Y[2]$ \\
\hline IP018 & 3 & JLNS & Peripheral blood & $46, X Y[2]$ \\
\hline IP030 & 4 & JLNS & Peripheral blood & $46, X Y[30]$ \\
\hline IP042 & 16 & ALS & Peripheral blood - erythroblasts & $46, X Y[20]$ \\
\hline IP052 & 12 & ALS & Peripheral blood - erythroblasts & $46, X Y[20]$ \\
\hline IP053 & 28 & ALS & Peripheral blood - erythroblasts & $46, X Y[20]$ \\
\hline IP021 & 3 & No & Peripheral blood & Without metaphases \\
\hline IP029 & 5 & No & Peripheral blood & $46, \mathrm{XX}[20]$ \\
\hline IP035 & 5 & No & Peripheral blood & $46, \mathrm{XX}[30]$ \\
\hline IP038 & 25 & No & Peripheral blood & $46, X Y, \operatorname{inv}(9)(p 12 q 13)[20]$ \\
\hline IP048 & 5 & No & Peripheral blood & $46, X Y[20]$ \\
\hline IP054 & 14 & No & Peripheral blood - erythroblasts & $46, X X[23]$ \\
\hline IP055 & 20 & No & Peripheral blood - erythroblasts & $46, X Y[20]$ \\
\hline IP046 & 21 & No & Peripheral blood - erythroblasts & $46, X Y[20]$ \\
\hline
\end{tabular}


Table 1 - Cont

\begin{tabular}{lcccc}
\hline Patient number & Passage & Disease & Source & Karyotype \\
\hline IP041 & 12 & No & Wharton's jelly & 46,XY[20] \\
IP043 & & No & Wharton's jelly & Without metaphases \\
IP044 & 24 & No & Wharton's jelly & 47,XY,+12[13] \\
IP045 & 13 & No & Wharton's jelly & 92,XXXX[20] \\
IP047 & 14 & No & Wharton's jelly & $46, X Y[20]$ \\
IP056 & No & Wharton's jelly & $46, X Y, a d d(14)(q 32)[13]$ \\
IP057 & No & Wharton's jelly & $48 \sim 92, X X X X[14]$ \\
IP058 & 17 & No & Wharton's jelly & 34 47,XY,+12[20] \\
IP059 & 34 & No & Wharton's jelly &
\end{tabular}

analyses in relation to the source are showed at Figure 2. This evaluation was performed only in the nondisease group, since among the individuals with diseases, there was no difference in the source (in all these cases, the source was peripheral blood). Samples without metaphases and less than 20 normal metaphases were excluded from this count.

\section{Influence of passage numbers}

The mean number of passages of the samples that presented clonal alterations was different from that of samples that presented with normal karyotypes.

In samples derived from peripheral blood, the mean number of passages of cells with a normal karyotype was 9.18 (standard deviation $=6.93$ ), while that of abnormal cells was 18.5 (standard deviation=8.67). The medians were also different: 6 for normal cells and 20 for altered cells.

Samples derived from Wharton's jelly also showed differences in the mean passage number. The passage number of samples with normal cells was 18.3 (standard deviation=7.58), and the passage number of samples with altered cells was 22.2 (standard deviation=7.13). The medians were also different: 14 for normal cells and 23 for altered cells.

\section{Karyotype before and after reprogramming}

It was possible to perform karyotype analysis before and after reprogramming in two samples of mesenchymal stem cells derived from Wharton's jelly. Each mesenchymal cell sample generated two iPSC samples (Table 2). A difference was observed between the results found before and after reprogramming in the two samples.

\section{Discussion}

The following chromosomal alterations were observed in this study: acentric fragments, chromosomal fusions, premature centromere divisions, double minutes, radial figures, ring chromosomes, polyploidies, inversions and trisomies. Both the disease and nondisease groups presented alterations. In the disease group, alterations were only observed in LQTS type 2 samples. In the nondisease group, the samples derived from Wharton's jelly presented both numerical and structural alterations, while the samples derived from blood presented only structural alterations.

Trisomy 12 was observed in two samples, which is concerning because according to Ben-David et al. (2014), in cell culture, this alteration may result in an increased cell proliferation rate and, consequently, increased in vivo tumorigenicity, inducing teratomas. In addition, lineages with this alteration behave differently from those with a normal karyotype, showing a different sensitivity in drug tests (Ben-David et al., 2014). Therefore, the presence of trisomy 12 could be cause for exclusion of such samples for use in both transplants and disease models.

Other changes observed may be markers of oncogenesis, such as a tetraploid karyotype, which has been described in the transition from premalignant to malignant disease, suggesting that duplication of the genome may be a driver of tumorigenesis (Dewhurst et al., 2014). Furthermore, the presence of additional material on chromosome 14 was observed in one of our samples. Changes in 14q32, such as translocations and additional material, are observed in patients with lymphomas and lymphocytic leukemias (Chen et al., 2016; Veloza et al., 2019).

Double minutes are small, acentric and paired structures and were detected in a sample from a patient with LQTS type 2 . The presence of dmins has been observed in patients with myelodysplastic syndrome, acute myelogenous leukemia or chronic myelomonocytic leukemia, and amplification of the MYC and MLL genes is observed in almost all cases (Huh et al., 2016). Future research with complementary molecular techniques could clarify whether the dmins observed after iPSC reprogramming could be copies of some of the genes used for reprogramming. The same sample also exhibited ring chromosomes in $6.7 \%$ of cells. Ring chromosomes arise after deletion or shortening of telomeric regions. Interestingly, shortening of telomeres associated with oxidative stress may cause genetic imbalances associated with ion channel defects in patients with LQTS (Yeh and Wang, 2016). Therefore, the presence of ring chromosomes may be a marker of LQTS, which is characterized by changes in sodium $\left(\mathrm{Na}^{+}\right)$and potassium $\left(\mathrm{K}^{+}\right)$ion channels (Nogueira et al., 2011). Nikitina et al. (2018) also reported the presence of ring chromosomes in iPSCs derived from skin fibroblasts; however, in this case, this chromosomal alteration was present before reprogramming because it was constitutional.

Chromosome 3 inversion, observed in a patient with LQTS, has previously been observed in patients with myelodysplastic syndrome and is often accompanied by alterations in other chromosomes. This inversion may be an indicator of evolution to acute myeloid leukemia and is 


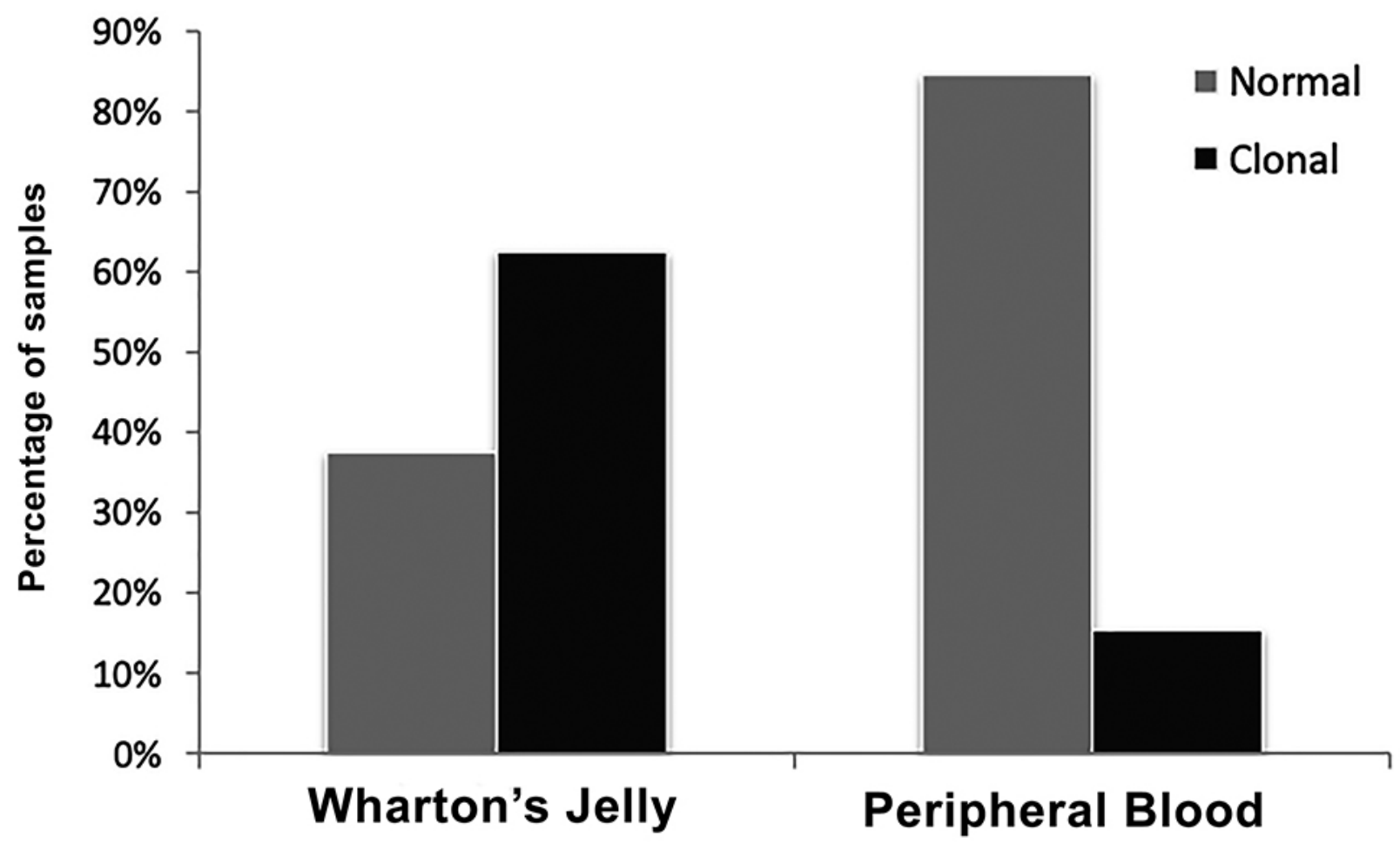

Figure 2 - Percentage of cases in the nondisease group with normal results x clonal alteration in different sources.

Table 2 - Cytogenetic result before (mesenchymal) and after (iPSCs) reprogramming.

\begin{tabular}{|c|c|c|c|}
\hline Mesenchymal sample & Mesenchymal result & $\begin{array}{c}\text { iPSC } \\
\text { samples }\end{array}$ & $\begin{array}{l}\text { iPSC } \\
\text { results }\end{array}$ \\
\hline \multirow{2}{*}{ ME310 } & \multirow{2}{*}{ 92,XXXX[4]/46,XX[29] } & IP045 & $92, \mathrm{XXXX}[20]$ \\
\hline & & IP057 & 48 92,XXXX[14] \\
\hline \multirow{2}{*}{ ME311 } & \multirow{2}{*}{$46, X Y[18]$} & IP044 & $47, \mathrm{XY},+12[13]$ \\
\hline & & IP058 & $34 \sim 47, \mathrm{XY}+12[20]$ \\
\hline
\end{tabular}

therefore considered a chromosomal alteration associated with poor prognosis (Cui et al., 2011; Sun et al., 2011).

Chromosome 9 inversion, observed in one of our samples, is an inherited variant in 0.8 to $2 \%$ of the world population (Lee et al., 2010) and is not necessarily associated with neoplastic diseases.

Regarding the when alterations appear during cultivation, our results show that clonal alterations can be present in several passages because they were detected from P6 to P34. However, in samples analyzed from different donors, we observed that changes were more frequent in the late passages, as demonstrated by the Mann-Whitney nonparametric statistical test ( $p=0.003$ ). The median number of passages of samples with alterations was 23 , and the median number of passages of samples with normal results was six. Our results, together with data from the literature (Mayshar et al., 2010), show that aberrations can emerge in the initial passages with low frequency and can be detected only in the late passages. These data confirm the importance of cytogenetic tests during the cultivation of iPSCs, even if the cells have been cultivated for only a few passages.

Five of nine samples generated from Wharton's jelly showed chromosomal aberrations. The only aberration found in the samples generated from peripheral blood was chromosome 9 inversion, which, as previously mentioned, is a variant commonly found in the population. Even when considering that chromosome 9 inversion may have developed during culture of iPSCs obtained from peripheral blood, we observed a significant difference between cells obtained from different sources (Fischer's exact test, $\mathrm{p}=0.017$ ), demonstrating an association between the source and the frequency of alterations. In this study, a greater frequency of chromosomal aberrations was observed in cells derived from Wharton's jelly than in cells derived from peripheral blood.

\section{Conclusion}

This study showed that, in iPSCs, there is a possibility that chromosomal aberrations, both clonal $(26.4 \%$ of our samples) and nonclonal (20.5\% of our samples), will emerge in several passages; these aberrations were detected from P6 to P34 and were more common in later passages.

After reprogramming and cultivation, some abnormal clones can emerge, as in the ME311 case, or be selected and generate a completely altered lineage, as in the ME310 case.

The following clonal chromosomal aberrations were detected in this study: chromosome 12 trisomy, which was 
previously reported in the literature; chromosome 3 inversion; chromosome 9 inversion; additional material on 14q; ring chromosomes; marker chromosomes; and dmins, which are being described now.

Many important chromosomal aberrations were detected, which demonstrated that G-banding is very useful for evaluation of genetic instability and has important repercussions for the application of iPSC lines.

\section{Acknowledgments}

This work was supported by MCTI/CNPq/MS (Grant number 404656/2012-9), FAPERJ, DECIT and in part by the Coordenação de Aperfeiçoamento de Pessoal de Nível Superior - Brasil (CAPES) - Finance Code 001.

\section{Conflicts of interest}

The authors declare that there is no conflict of interest that could be perceived as prejudicial to the impartiality of the reported research.

\section{Author contributions}

IMV wrote the paper and assembled the karyograms; IMV and TB carried out the chromosomal analysis of karyograms and karyotypes; THKB, DSS, FCPM, JFV and FG prepared the cells, carried out cell cultivation and reprogramming of iPSCs and reviewed the paper; TB, CLKR and ACS helped to draft the manuscript and reviewed the paper. PRSB coordinated the study. All of the authors read and approved the manuscript.

\section{References}

Ambrosio C, Zomer H, Vidane A and Gonçalves N (2015) Mesenchymal and induced pluripotent stem cells: general insights and clinical perspectives. Stem Cells Cloning Adv Appl 8:125.

Ben-David U, Arad G, Weissbein U, Mandefro B, Maimon A, Golan-Lev T, Narwani K, Clark AT, Andrews PW, Benvenisty $\mathrm{N}$ et al. (2014) Aneuploidy induces profound changes in gene expression, proliferation and tumorigenicity of human pluripotent stem cells. Nat Commun 5:4825.

Ben-David U and Benvenisty N (2012) Analyzing the genomic integrity of stem cells. In: StemBook. Harvard Stem Cell Instite, Cambridge, MA.

Ben-David U and Benvenisty N (2011) The tumorigenicity of human embryonic and induced pluripotent stem cells. Nat Rev Cancer 11:268-277.

Borgonovo T, Vaz IM, Senegaglia AC, Rebelatto CLK and Brofman PRS (2014) Genetic evaluation of mesenchymal stem cells by G-banded karyotyping in a cell technology center. Rev Bras Hematol Hemoter 36:202-207.

Catalina P, Cobo F, Cortés JL, Nieto AI, Cabrera C, Montes R, Concha A and Menendez P (2007) Conventional and molecular cytogenetic diagnostic methods in stem cell research: A concise review. Cell Biol Int 31:861-869.

Chen W, Miao Y, Wang R, Wu Y, Qiu H, Xu W, Li J, Fan L and Xu $\mathrm{X}(2016) \mathrm{t}(14 ; 18)(\mathrm{q} 32 ; \mathrm{q} 21)$ in chronic lymphocytic leukemia patients: Report of two cases and a literature review. Oncol Lett 12:4351-4356.

Cui W, Sun J, Cotta CV, Medeiros LJ and Lin P (2011) Myelodysplastic syndrome with $\operatorname{inv}(3)(\mathrm{q} 21 \mathrm{q} 26.2)$ or $\mathrm{t}(3 ; 3)(\mathrm{q} 21 ; \mathrm{q} 26.2)$ has a high risk for progression to acute myeloid leukemia. Am J Clin Pathol 136:282-288.

Dewhurst SM, McGranahan N, Burrell RA, Rowan AJ, Grönroos E, Endesfelder D, Joshi T, Mouradov D, Gibbs P, Ward RL et al. (2014) Tolerance of whole- genome doubling propagates chromosomal instability and accelerates cancer genome evolution. Cancer Discov 4:175-185.

Garcia-Martinez J, Bakker B, Schukken KM, Simon JE and Foijer F (2016) Aneuploidy in stem cells. World J Stem Cells 8:216-222.

Gubert F, Vasques JF, Cozendey TD, Domizi P, Toledo MF, KasaiBrunswick TH, Hochman-Mendez C, Campos Junior M, Zembrzuski VM, Loureiro MPS et al. (2019a) Generation of patient-specific pluripotent induced stem cell line UFRJi007-A from a Brazilian familial amyotrophic lateral sclerosis patient. Stem Cell Res 39:101490.

Gubert F, Vasques JF, Cozendey TD, Domizi P, Toledo MF, KasaiBrunswick TH, Loureiro MPS, Lima JMB, Gress CH, Cabello GMK et al. (2019b) Generation of four patient-specific pluripotent induced stem cell lines from two Brazilian patients with amyotrophic lateral sclerosis and two healthy subjects. Stem Cell Res 37:101448.

Huh YO, Tang G, Talwalkar SS, Khoury JD, Ohanian M, Bueso-Ramos CE and Abruzzo LV (2016) Double minute chromosomes in acute myeloid leukemia, myelodysplastic syndromes, and chronic myelomonocytic leukemia are associated with micronuclei, MYC or MLL amplification, and complex karyotype. Cancer Genet 209:313-320.

Kasai-Brunswick TH, Silva dos Santos D, Ferreira RP, Araujo DS, Dias GM, Coutinho JLA, Cruz FESF, Sternick EB, Gubert F, Oliveira JCG et al. (2018) Generation of patient-specific induced pluripotent stem cell lines from one patient with Jervell and Lange-Nielsen syndrome, one with type 1 long QT syndrome and two healthy relatives. Stem Cell Res 31:174-180.

Lamm N, Ben-David U, Golan-Lev T, Storchová Z, Benvenisty N and Kerem B (2016) Genomic instability in human pluripotent stem cells arises from replicative stress and chromosome condensation defects. Cell Stem Cell 18:253-261.

Lee S-G, Park TS, Lim G, Lee K-A, Song J and Choi JR (2010) Constitutional pericentric inversion 9 and hematological disorders: A Korean tertiary institution's experience over eight years. Ann Clin Lab Sci 40:273-277.

Mayshar Y, Ben-David U, Lavon N, Biancotti J-C, Yakir B, Clark AT, Plath K, Lowry WE and Benvenisty N (2010) Identification and classification of chromosomal aberrations in human induced pluripotent stem cells. Cell Stem Cell 7:521-531.

McGowan-Jordan J, Simons A and Schmid M (2016) ISCN 2016 an international system for human cytogenomic nomenclature. 2016th edition, Karger, Basel, Switzerland, 139 p.

Mesquita FCP, Arantes PC, Kasai-Brunswick TH, Araujo DS, Gubert F, Monnerat G, Silva dos Santos D, Neiman G, Leitão IC, Barbosa RAQ et al. (2019) R534C mutation in hERG causes a trafficking defect in iPSC-derived cardiomyocytes from patients with type 2 long QT syndrome. Sci Rep 9:19203.

Moralli D, Yusuf M, Mandegar MA, Khoja S, Monaco ZL and Volpi EV (2011) An improved technique for chromosomal analysis of human ES and iPS cells. Stem Cell Rev Rep 7:471-477.

Moretti A, Bellin M, Welling A, Jung CB, Lam JT, Bott-Flügel L, Dorn T, Goedel A, Höhnke C, Hofmann F et al. (2010) Patient-specific induced pluripotent stem-cell models for long-QT syndrome. N Engl J Med 363:1397-1409.

Nikitina TV, Menzorov AG, Kashevarova AA, Gridina MM, Khabarova AA, Yakovleva YS, Lopatkina ME, Kizilova EA, Vasilyev SA, Serov OL et al. (2018) Generation of two iPSC lines (IMGTi001-A and IMGTi001-B) from human skin fibroblasts with ring chromosome 22. Stem Cell Res $31: 244-248$.

Nogueira RGB, Rebello ES, Rebello APS, Sotomaior VS and Raskin S (2011) Síndrome do QT longo. Estud Biol 32:105-110. 
Sun J, Konoplev SN, Wang X, Cui W, Chen SS, Medeiros LJ and Lin P (2011) De novo acute myeloid leukemia with inv(3) (q21q26.2) or $\mathrm{t}(3 ; 3)(\mathrm{q} 21 ; \mathrm{q} 26.2)$ : A clinicopathologic and cytogenetic study of an entity recently added to the WHO classification. Mod Pathol 24:384-389.

Taapken SM, Nisler BS, Newton MA, Sampsell-Barron TL, Leonhard KA, McIntire EM and Montgomery KD (2011) Karyotypic abnormalities in human induced pluripotent stem cells and embryonic stem cells. Nat Biotechnol 29:313-314.
Veloza L, Ribera-Cortada I and Campo E (2019) Mantle cell lymphoma pathology update in the 2016 WHO classification. Ann Lymphoma 3:3.

Yeh J-K and Wang C-Y (2016) Telomeres and telomerase in cardiovascular diseases. Genes (Basel) 7:58.

Associate Editor: Alysson Muotri

License information: This is an open-access article distributed under the terms of the Creative Commons Attribution License (type CC-BY), which permits unrestricted use, distribution and reproduction in any medium, provided the original article is properly cited. 